Fermilab Proposal No.4k5

Scientific Spokesman: V. Z. Peterson

Department of Physics

University of Hawaii

Honolulu, Hawaii

Telephone: $\quad(808) 948-7391$

\title{
BUBBLE-CHAMBER STUDY OF DIMUON PRODUCTION BY NEUTRINOS \\ USING THE PHASE-2 EMI AND A DICHROMATIC BEAM
}

R. J. Cence, F. A. Harris, S. I. Parker, M. W. Peters, V. Z. Peterson and V. J. Stenger University of Hawaii

and

o. I. Dahl, A. Barbaro-Galtieri, P. H. Eberhard, G. R. Lynch, J. P. Marriner and M. L. Stevenson University of California, Lawrence Berkeley Laboratory

October 17, 1975 


\section{OUTLINE}

BUBBLE-CHAMBER STUDY OF DIMUON PRODUCTION BY NEUTRINOS USING THE PHASE-2 EMI AND A DICHROMATIC BEAM

Page

Summary . . . . . . . . . . . . . . . . . . . $i$

I. InTRODUCTION. . . . . . . . . . . . . 1

II. EXPERIMENTAL ASPECTS............... 5

(A) Improved EMI (EMI-IIa) . . . . . . . . . 5

(B) Choice of Light Neon-Hydrogen Liquid . . . . 12 ( $30 \%$ neon atoms)

(C) Dichromatic Beam (the Baltay two-horn design). . 14

(D) Estimated Yields for 200K Picture Exposure . . 16

III. DILEPTON PRODUCTION . . . . . . . . . . . 18

IV. DIFFRACTIVE PRODUCTION OF VECTOR PARTICLES. . . . 20

V. NEUTRAL CURRENT INTERACTIONS. .......... 23

VI. OTHER PHYSICS ASPECTS OF THE EXPERIMENT . . . . . 26

VII. RELATIONSHIP TO OTHER NEUTRINO EXPERIMENTS . . . . 27

References . . . . . . . . . . . . . . . 29

Figures .................... 32 
We propose to examine in detail, using the 15-foot bubble chamber and an improved ("Phase II") EMI, the characteristics of "dimuon" events (1) produced by neutrinos. A light neon-hydrogen filling ( $30 \%$ neon atoms) provides adequate target mass, good track measurements, and high detection efficiency for photons and electrons. Thus $e-\mu$ and $e-e$ dileptons, as well as dimuons, can be observed with good efficiency.

We estimate a yield of 100 detected dimuons in a $200 \mathrm{~K}$ picture exposure. We assume $400 \mathrm{GeV}$ operation, $1 \times 10^{13}$ protons/pulse, and a two-horn dichromatic beam ${ }^{(2)}$ focusing $100 \pm 10 \mathrm{GeV} / \mathrm{c}$ mesons. If dimuons are made by neutrinos $\geq 30 \mathrm{GeV}$, then the yield from this dichromatic beam is about half the yield from a wide-band beam. Knowledge of neutrino energy is important in dimuon analysis.

An improved two-plane EMI, as proposed by the UH-LBL group, would provide about $1 \mathrm{~kg} / \mathrm{cm}^{2}$ absorption thickness for particles above $10 \mathrm{GeV} / \mathrm{C}$, this ensuring excellent dimuon identification. Another proposed EMI improvement, the Internal Picket Fence (IPF), is designed to eliminate EMI random background (mainly neutrino-induced in the internal coil-absorber). This should greatly simplify EMI analysis and reduce the misidentification of low momentum hadrons as muons. Thus we also expect improved efficiency for identification of neutral current events and slow (wide-angle) muons with this "Phase II" EMI. 
This exposure should yield charged-current (CC) and neutral-current (NC) events as follows:

$$
\begin{array}{lll}
E_{V}=35 \mathrm{GeV} & 9000 & \frac{N C}{2250} \\
E_{V}=92 \mathrm{GeV} & 1000 & 250
\end{array}
$$

With improved EMI muon identification and reduced background, we expect to be able to determine $R=N C / C C$ within low systematic errors.

The bubbie-chamber/EMI/dichromatic beam is also ideally suited to study the diffractive production of vector mesons $(\rho, \omega, \phi, A)$ by neutrinos. The theoretical importance of these processes in determining the characteristics of the weak interaction and probable connections with the "high-y anomaly" and dimuon production has been emphasized recentiy. $(3,4,5)$ We would first search for CC events of the type

$$
\begin{aligned}
& \nu p \rightarrow \mu^{-} p \rho^{+},\left(\rho^{+} \rightarrow \pi^{+} \pi^{0}\right) \\
& \nu p \rightarrow \mu^{-} p A_{1}^{+},\left(A_{1}^{+}+\pi^{+} \rho^{o}\right)
\end{aligned}
$$

and NC events of the type

$$
\begin{aligned}
& \nu p \rightarrow \nu p \rho^{0},\left(\rho^{0} \rightarrow \pi^{+} \pi^{-}\right) \\
& \nu p \rightarrow \nu p \phi, \quad\left(\phi \rightarrow K^{+} K^{-}\right)
\end{aligned}
$$

as evidence of diffractive productive of vector mesons.

A continuing search for "charm" will be made using single strange particle events $(\Delta S=-\Delta Q)$ as a signature. We expect about 350 single $\Lambda^{\prime} s$ in this sample. 
BUBBLE-CHAMBER STUDY OF DIMUON PRODUCTION BY

NEUTRINOS USING THE PHASE-2 EMI AND A DICHROMATIC BEAM

\section{INTRODUCTION}

The most interesting result of the first round of neutrino counter experiments at Fermilab has been the discovery of "dimuon events" by the HPWF collaboration. (1) The signal appears to be well above meson decay backgrounds and thus is interpreted as "directly produced." Despite very little detailed knowledge of the hadronic states accompanying dimuon events, the evidence from the fast muons and the total hadronic signal (calorimeter output) has been interpreted as sufficient to rule out intermediate vector bosons or heavy leptons as sources of the second muon. Instead, it is argued that the dimuon events, in association with a "high-y anomaly" in deep inelastic scattering, is sufficient to propose a new particle emerging as the hadronic vertex; i.e.,
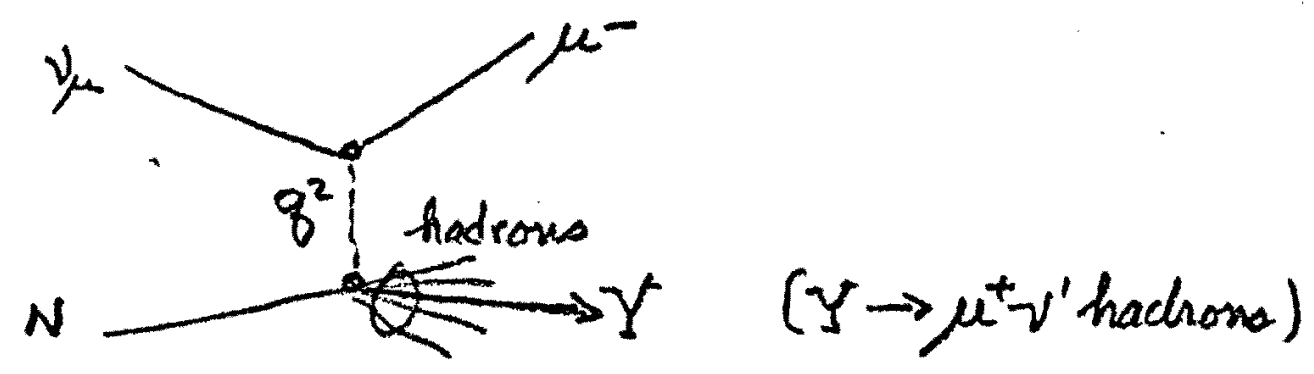

where the new particle, $Y+\mu^{+}+\nu_{u}+$ hadrons, must carry a new quantum number which might be associated with "charm." Every experimental effort should be made to learn more about such events, and in particular the hadronic final state should be studied in detail. The bubble chamber is a natural 
detector for studying the hadronic vertex, but present exposures suffer from problems of low rate (dimuons occur at $1 \%$ of single muon events over $30 \mathrm{GeV}$ ) and dimuon identification (the present External Muon Identifier (EMI-I) permits hadron punch-through at the $4 \%$ leve1). The present proposal assumes that EMI-II (an improved EMI with external absorber and a second plane)(6) will remedy this latter problem, and that the higher energy (400 GeV) accelerator operation at increased beam intensity will make tolerable the rate problem with neon-hydrogen fillings. With $400 \mathrm{GeV}$ high intensity running even narrow-band beams are practical for dimuon studies.

A related physics objective of this proposal is to study the diffractive production of vector particles which may be related to the occurrence of dimuons. $(3,4,5,15)$ The observed characteristics of dimuon events, (1) such as the chargeasymmetry between negative and positive muons and the spread in invariant mass of dimuons, have stimulated theoretical papers $(3,4,5,15)$ which propose that vector mesons possessing quantum numbers of the weak interaction may be produced by a "vector meson dominance intermediate $F *$ mechanism" as follows:

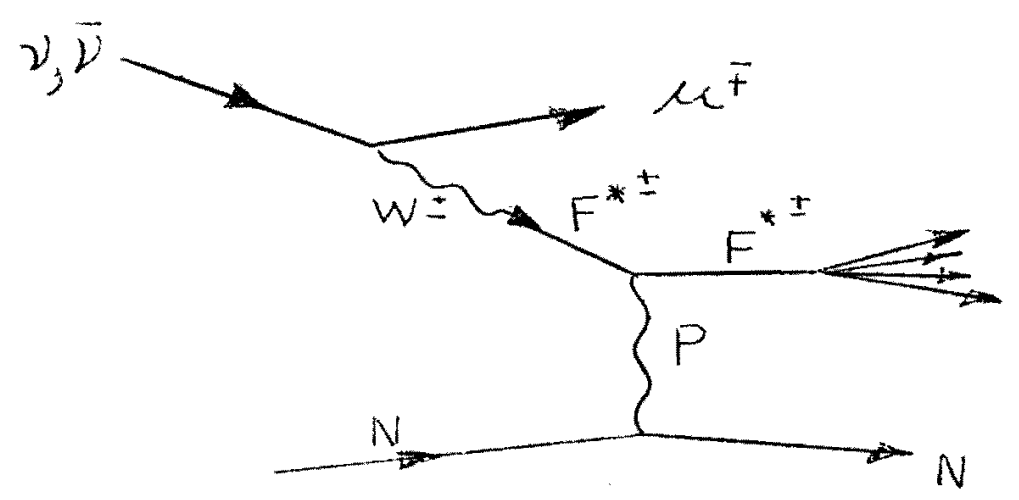


Barger, Weiler and Phillips $(3)$ deduced that the $v=x y$ and momentum distributions of the fast $\mu^{-}$in the $\mu^{+} \mu^{-}$dimuon events are consistent with this model. (3) Furthermore, this process seems to account for a substantial part of the cross section at smal1 $x$ and large $y$ (i.e., the "high-y anomaly"). Dimuon production is then accounted for by the proposal(7) that a new "charmed hadron" $Y \rightarrow \mu^{+} v_{\mu}+$ hadrons is contained within "X" above.

We propose to study diffractive processes using the bubble-chamber technique to first search for the "established" vector mesons ( $\rho, A, \phi, \psi$, etc.) in various combinations of charged and neutral hadrons. Obviously, the dimuon candidates selected by EMI-II will receive special attention. The light neon-hydrogen mixture is optimum for investigating exclusive reactions. Since a missing neutrino may be involved in even charged-current events (i.e., $\left.Y \rightarrow \mu^{+} v_{\mu} x\right)$, it is important to have knowledge of the incident neutrino energy; hence the dichromatic beam.

In the course of these special investigations we will be able to analyze about 10,000 charged current (single muon) events and 2500 neutral current (a11 hadron) events from "normal" inelastic scattering. The $x$ - and $y$-distributions for these events, particularly charged current events with wideangle muons and all neutral current events, are still not well determined by existing experiments. 
A major limitation of the present bubble-chamber/EMI-I hybrid system for neutral current studies, namely the random background of neutrino-induced events in coils and absorber which causes some slow hadrons to look like muons, should be eliminated in EMI-II by the Internal Picket Fence (IPF) ${ }^{(16)}$ (see Section II). We expect this to bring greatly improved reliability in the selection of $C C$ and NC events. Knowledge of incident neutrino energies is extremely valuable, especially for neutral current events.

We also expect to analyze events containing strange particles, especially single strange particle which signify $\Delta S=-\Delta Q$ processes, in the general search for "charm." It is rather surprising that the single, low-energy event found by the BNL group $(8)$ has not yet been confirmed by the much more sensitive Fermilab bubble-chamber experiments. The present proposed experiment would have certain advantages (i.e., better muon detection, electron/photon detection, knowledge of incident neutrino energy) than existing 15 -foot chamber exposures.

The following sections detail the special experimental aspects of this proposal and their consequences for the three major physics goals:

(1) dimuon production

(2) diffractive production of vector mesons

(3) improved detection of neutral current events. 
II. EXPERIMENTAL ASPECTS

Since the general features of the Fermilab neutrino bubble-chamber program (including the single-plane EMI) are by now well known, we will limit ourselves to the new features of this proposal, namely: (a) an improved EMI ("EMI-II")' with external absorber and a second plane, plus the Internal Picket Fence; $($ (b) the choice of light neon-hydrogen ( $30 \%$ neon atoms) bubble-chamber liquid for optimum targetdetector properties; (c) use of a dichromatic beam, made practicable by $400 \mathrm{GeV}$ operation and increased proton intensity; and (d) significant yields of interesting types of events (e.g., dimuons).

In a separate document submitted to the Fermilab Directorate, the UH-LBL "EMI group" has described in some detail its proposals for EMI-II; i.e., improvement of the present EMI, for future use as a general facility. Both short-range (IPF and 1 imited second plane) and long-range (full second-plane and additional area coverage) are proposed. only the short-range program is needed for this experiment, and this should be achievable on the same time-scale as the new two-horn dichromatic beam.

(A) Improved EMI (EMI-IIa). The original EMI proposal (9) envisaged a two-plane EMI with area coverage of $50 \mathrm{~m}^{2}$ and absorber between planes; the two-plane feature was to measure the direction of outgoing prongs and to reduce background 
by requiring time coincidences between the two planes. This proposal was made prior to the discovery of neutral currents and dimuons. Our $3 \mathrm{GeV}$ Bevatron test of the EMI principle(10) appeared to show that one-plane was "good enough" for detecting $96 \%$ of single muons while rejecting $95 \pm 1 \%$ of the pions. Accordingly, we scaled down our proposal to a one-plane EMI of $30 \mathrm{~m}^{2}$ which was later reduced to $24 \mathrm{~m}^{2}$ as module costs exceeded initial estimates. This scaled-down EMI became operational in time to be useful during the "first round" 15-foot bubble-chamber experiments which had been expected to be "bare-bubble-chamber" exposures.

Our experience in analyzing a portion of the $68 \mathrm{~K}$ pictures of the E-45 experiment (neutrinos on hydrogen, horn beam at $300 \mathrm{GeV})$, plus a smaller fraction of E-28 film $(22 \%$ neonhydrogen, horn beam, $300 \mathrm{GeV}$ ), has confirmed most of the initial predictions for single-muon EMI detection.

(a) "Muons" are defined as tracks falling inside a "muon ellipse" containing $96 \%$ of the muons.

(b) The initial absorber (coils plus zinc) permits about $4 \%$ of all hadrons to "punch-through" simulating muons; however, higher momentum hadrons punch through more often.

(c) The measured background of "hits" in the EMI (about $4 \mathrm{~m}^{-2}$ per $10^{13}$ protons on target from neutrino interactions in the downstream coil and absorbers is somewhat higher than expected. A $1-\mathrm{m}^{2}$ "second-plane" test ${ }^{(11)}$ shows that 
most of this signal is due to "soft" radiation which is eliminated if time coincidences are required.

(d) The limited $24 \mathrm{~m}^{2}$ area coverage covers most of the wide-band beam produced muons from CC events; however, slow muons from interesting high-y (1arge energy transfer to the hadron) events are missed. The overall efficiency for detecting the single muon events is $80 \%$ for the wide-band beam.

Detection of neutral currents places a much greater demand upon the EMI since it must certify that none of the visible prongs is a muon (or at least that none of the negative prongs is a muon). The limitation of random background accidentals (which make hadrons look like muons) is serious for NC events. We have upgraded the present EMI since the early E-45 runs by adding more internal absorber; preliminary data from the E-28 experiment show clearly that there is less punch-through, but random accidentals are still serious for low momentum tracks.

Searching for dimuons at the $1 \%$ level is clearly almost out of the question for an EMI with $4 \%$ hadron punch-through. Our E-45 data show two dozen dimuon candidates (about $4 \%$ of the $C C$ events) after selecting only time-coincidence tracks. We would expect to see 2.7 dimuons (1\% of CC events above $30 \mathrm{GeV})$. The ratio of,+--- , and ++ dimuon candidates is consistent with the charge ratio of positive and negative hadrons able to punch through. We are pursuing the analysis 
of these events to find the "most probable dimuons," but the analys is cannot be considered an independent proof of dimuon production.

Two-planes and external absorber

Clearly, therefore, we need additional absorber in substantial amounts to detect dimuons with certainty. The present internal coil-absorber configuration is shown in Fig. 1 ; the average neutrino secondary passes through 3.5 pion absorption lengths $\left(L_{\pi} \simeq 150 \mathrm{gm} / \mathrm{cm}^{2}\right.$ of copper or zinc) in reaching the EMI modules.

The probability of hadron "punch-through" (to land within the "muon ellipse" as a single "hit") is somewhat difficult to calculate reliably since it involves following a hadron cascade through many interaction lengths and taking into account multiple-scattering, magnetic field effects, etc. The simplest model appears to assume the "punch-through" consists entireiy of non-interacting hadrons with an effective absorption length, $\lambda_{a}^{\prime}(p)$; a function of momentum. If we ascribe all E-45 dimuon candidates to hadron "punch-through," we obtain $\lambda^{\prime}(p)$ as follows (using $x=260 \pm 60 \mathrm{gm} / \mathrm{cm}^{2}$ for $E-45$ data).

Table II -1

E-45 Punch-through Results

Hadron Momentum $<5 \mathrm{GeV} / \mathrm{c}$ $5-10$ $>10$ A11

$\begin{array}{r}\text { Extrapo } \\ 169 \\ 59 \\ 24 \\ \hline 252\end{array}$

252

\section{Punch-} through

5

4

$-2$ 11 $-x / \lambda^{\prime}$ e $\lambda_{a}^{\prime}$ $.030 \quad 74 \mathrm{gm} / \mathrm{cm}^{2}$ $.068 \quad 97$ $.083 \quad 104$ $.044 \quad 83$ 
These results appear to be considerably different from the CERN Monte Carlo predictions, (13) which yield $\lambda^{\prime}(3 \mathrm{GeV})=$ $136 \mathrm{gm} / \mathrm{cm}^{2}$ and $\lambda^{\prime}(15 \mathrm{GeV})=273 \mathrm{gm} / \mathrm{cm}^{2}$. This is a very important discrepancy and better statistics are needed (and will soon be available from E-28 data taken with 100 to 200 $\mathrm{gm} / \mathrm{cm}^{2}$ additional zinc). Tentatively, we assume $\lambda^{\prime}(p>10$ $\mathrm{GeV} / \mathrm{C})=135 \mathrm{gm} / \mathrm{cm}^{2}$, the standard pion absorption length, (14) for the external absorber.

Assuming that we wish to reduce the observed punchthrough of a high-momentum $(p>10 \mathrm{GeV} / \mathrm{c})$ hadron to well below $1 \%$ (say, $0.1 \%$ ), the additional thickness $\Delta x$ required is given by:

$$
\begin{aligned}
& (.083) \mathrm{e}^{-\Delta x / \lambda^{\prime}}=.001 \quad\left(\lambda^{\prime}=135 \mathrm{gm} / \mathrm{cm}^{2}\right) \\
& \therefore \Delta x=4.42 \lambda^{\prime}=600 \mathrm{gm} / \mathrm{cm}^{2}(84 \mathrm{~cm} . \text { zinc }) .
\end{aligned}
$$

The area of such absorber required for dimuon detection is also an important parameter. The HPWF dimuons seem to be produced by neutrinos above $30 \mathrm{GeV}$ and the slower muons (positives) generally exceed $10 \mathrm{GeV}$ with a few between 5 and $10 \mathrm{GeV}$. Fig. 2 shows the hit pattern of all tracks from fiducial volume neutrino events in our E-45 sample for momenta above $10 \mathrm{GeV} / \mathrm{c}$. A rectangular area $3 \mathrm{~m}$. high by $4 \mathrm{~m}$. wide at the present EMI plane would intercept all such prongs from CC events in E-45 (90\% of hadrons 5-10 GeV/C would also be included). We propose to install such an absorber (zinc) immediately behind EMI Plane I, as shown in Fig. 3. This 
absorber would weigh 80 tons. The second EMI plane of $12 \mathrm{~m}^{2}$ would be mounted directly behind the zinc wall. Details of construction and proposed operation are given in a separate facility proposal. (6)

We believe that this portion of the Phase-2 EMI would provide at least the same efficiency for dimuon detection as E-1A by eliminating punch-through. In fact, the geometrical coverage of the 15-foot bubble chamber plus this 2-plane EMI is greater than E-1A's coverage, as can be seen from Fig. 4 which superimposes E-Ia calorimeter and muon spectrometer outline on a plan view of the 15-foot bubble chamber/EMI.

of course the bubble chamber should be far superior to the calorimeter in detecting the final hadronic state, observing meson decays in flight, etc.

Internal Picket Fence (IPF)

Our EMI improvement proposal ${ }^{(6)}$ includes a "short-range" project to construct a "picket fence" of tubular gas proportional counters inside the bubble-chamber coils and just outside the bubble-chamber liquid on the downstream side (see Fig. 5). The IPF consists of 120 clusters of long cylindrical singlewire proportional chambers $(60$ clusters above the median plane, 60 below). Each cluster consists of 8 tubes, 2 layers of 4 tubes. Each tube is 1 -inch in diameter and 7.5 meters long. The projected width of each cluster is 4 inches. The outputs of all 8 counters are ganged, and the two layers of tubes provide backup if one counter fails. The major development 
problems consist of (a) "space-hardening" these counters to operate indefinitely in a vacuum without access and (b) maintaning the proper temperature range of gas proportional counter operation. Preliminary tests are now underway.

The advantage of the IPF is that it promises to provide a bubble-chamber event timing signal from the time-coincidence of two or more "selected" clusters. A "selected" cluster is one in which a neutrino event track projects to hit the cluster. Thus the IPF is operated as a hodoscope, and the IPF and EMI are analyzed only. for neutrino events. Each cluster's output is digitized in the same manner as the outputs from the EMI modules. The measured EMI module singles rate. is $4 \mathrm{hits} / \mathrm{m}^{2} / 10^{13} \mathrm{ppp}$, so we expect each cluster to count less than $0.60 / 10^{13} \mathrm{ppp}$. Thus accidental two-fold coincidences during a resolving time of 33 nsec. would be negligible.

The primary advantage of the IPF is to reduce the random background of EMI Plane-l hits due to neutrino interactions in the absorber/coils."These background hits in general will be out of time. We estimate that $\geq 98 \%$ of the present EMI hits (i.e., all but 1 or 2 of the 100 total) relating to a frame containing a valid bubble-chamber neutrino event are "background" in that they are not time-associated with the primary neutrino event.

The IPF promises to simplify EMI-BC analysis procedures, since at present all background hits must be considered 
candidates for track matching. The increased reliability in hadron/muon discrimination for lower momentum tracks (below about 5 GeV) will reduce uncertainties in selecting NC and CC events.

The IPF does not solve the problem of punch-through since hadronic punch-through will be in time coincidence; however, the "central area" $(3 \mathrm{~m}$. $\times 4 \mathrm{~m}$. covered by the second plane with thick external absorber) will reject hadrons and detect all but very slow muons. Outside the central area, the probability of labeling a wide-angle slow hadron as a "muon" will be radically decreased.

Thus, the two "short-range" EMI improvements, a thick external absorber and second plane plus an Internal Picket Fence, are essential to a high efficiency search for dimuons and for low background analysis of neutral current and diffractive production of vector mesons.

(B) Choice of Light Neon-Hydrogen Liquid $130 \%$ neon atoms). We strongly prefer a light neon-hydrogen mixture, about 30 atomic percent neon, for this experiment using the $B C-E M I$ hybrid system for the following reasons:

(1) $30 \%$ neon-hydrogen has high enough density $\left(\rho=0.4 \mathrm{gm} / \mathrm{cm}^{3}\right)$ so that neutrino events occur at a reasonable rate. (One neutrino event per 20 pictures; see II-D.) 
(2) $30 \%$ neon-hydrogen provides $90 \%$ photon conversion and good electron identification for events originating in the fiducial volume. (Our present E45 fiducial volume of $20 \mathrm{~m}^{3}$ has an average radius of $1.7 \mathrm{~m}$. and height of $2.8 \mathrm{~m}) \cdot 30 \%$ neon-hydrogen has a radiation length of $80 \mathrm{~cm}$. Thus we expect to see both photons from $80 \%$ of our pi-zeros.

(3) We need to make momentum measurements for charged particles with accuracies approaching that possible with bydrogen if we are to search for resonances in the hadronic final state! Thus we need an interaction length longer than the average track length. (30\% neon-hydrogen has a collision length of $250 \mathrm{~cm}$., as compared with an average track length of $170 \mathrm{~cm}$. for fiducial volume events. $\Delta p / p$ accuracies similar to hydrogen can be expected in $30 \%$ neon up to about $10 \mathrm{GeV} / \mathrm{c}$ which includes most of the hadrons from neutrino events.)

(4) Neutron detection depends both upon density and ability to see the proton recoil. For $30 \%$ neonhydrogen about $50 \%$ of the neutrons will interact within the chamber. Recoils can be seen to lower energies in $30 \%$ neon-hydrogen than in heavier neon-hydrogen mixtures. Another practical aspect is that the 15-foot chamber appears to work we 11 with $\sim 30 \%$ neon $(E-28$ ran at $22 \%$ neon concentration). Furthermore, we now have considerable experience in 
analyzing film taken with light neon. The "hydrogen-like" appearance of heavy particle tracks, plus the ability to recognize electrons and to detect electron-positron pairs, are qualitative reasons for preferring this mixture.

(C) Dichromatic Beam (the Baltay two-horn design). We propose to use the new two-horn dichromatic beam, designed by $C$. Baltay and $D$. Cohen (2) and scheduled to be ready by mid-1976. This improved dichromatic beam was designed specifically to be used with the 15-foot bubble chamber and to accommodate the constraints of the present horn mount and power supply and to fit into the present target tunnel. The solid angle acceptance of the first horn (150 uster) is considerably larger than that of the existing CalTech dichromatic beam (12 uster). The usefulness of the larger solid angle is greatest for lower momentum mesons since the average production angle decreases with momentum. At high momenta the collimating plug on axis between the first and second horn increasingly attenuates the total yield. Thus this two-horn focusing device has a neutrino event yield peaking at intermediate momenta $(100 \mathrm{GeV} / \mathrm{C})$.

We propose to use the two-horn dichromatic beam focused for a mean meson momentum of $100 \mathrm{GeV} / \mathrm{c}$. Thus the mean momentum of muon neutrinos from pion decay will be $36 \mathrm{GeV} / \mathrm{c}$ and the mean momentum of muon neutrinos from kaon decay will be $92 \mathrm{GeV} / \mathrm{c}$. (2)

The energy resolution of this dichromatic beam depends upon the meson momentum bandwith $\left(\Delta P_{0} / P_{0}= \pm 10 \%\right.$ or $\left.\pm 5 \%\right)$ and 
also the kinematics of meson decay. Kaon neutrinos have good energy resolution over the entire bubble chamber $\Delta E / E=\Delta P_{0} / P_{0}$ due to the large transverse momentum in $K \rightarrow \mu \nu$ decay. The energy spread of the pion-source neutrinos is larger over the entire bubble chamber, but by utilizing the known position of the neutrino interaction in the bubble chamber a neutrino energy resolution $\Delta E / E=\Delta P_{0} / P_{0}$ is also possible. Thus we assume $\Delta E / E=\Delta P_{0} / P_{0}$ from either pion or kaon neutrinos.

The ability to know that the incident neutrino has one of two discrete energies (within $\pm 5 \%$ or $\pm 10 \%$ ) is very important in analyzing dimuon and neutral current events. The high detection efficiency of the neon-hydrogen bubble chamber combined with the constraint of a dichromatic beam should drastically reduce the uncertainties in understanding rare events. For example, the assumption of a missing neutrino in dimuon event can be checked by comparing the calculated incident neutrino energy with dichromatic values.

The dichromatic beam has a spectrum shown in Fig. 6 which is taken from the Baltay-Cohen design. (2) The relative solid angle improvement of this design over the existing two-horn wide-band beam (WBB) is such that the yield of charged current events above 30 GeV neutrino energy is down by only a factor of two; i.e.,

$$
\left.\frac{\text { Dichromatic yield }}{\text { Wide-band yield }}=\frac{1}{2} \text {, (CC events, } E_{\nu}>30 \mathrm{GeV}\right) \text {. }
$$

This assumes $\Delta P_{0} / P_{0}= \pm 10 \%$. 
(D) Estimated Yields for $200 \mathrm{~K}$ Picture Exposure. Using the information on beam and detector summarized above, we next estimate the yield of neutrino events of various types. We assume $400 \mathrm{GeV}$ operation, $1 \times 10^{13}$ protons per pulse on target, mean meson energy of $100 \pm 10 \mathrm{GeV}$ for the dichromatic beam, and an exposure of 200,000 pictures.

Table II-2 below lists the estimated yields of events in various categories within a $20 \mathrm{~m}^{3}$ fiducial volume.

Table II -2

Estimated Yields of Various Categories of Neutrino Events (200K pix, $400 \mathrm{GeV}, 1 \times 10^{13} \mathrm{ppp}$ )

$\begin{array}{rrr} & \frac{E=36 \mathrm{GeV}}{} & \frac{E=92 \mathrm{GeV}}{1000} \\ \text { CC events (a11 types).... } & 9000 & 10 \\ \text { Dimuon events (1\%)... } & 90 & 250\end{array}$

Thus the total yield $(N C+C C)$ events is 12,500 in $200 \mathrm{~K}$ pictures, or one neutrino event every 16 pictures. The above table is based upon a total ćross section (for CC events) of $\sigma=0.8 \mathrm{E}$ $10^{-38} \mathrm{~cm}^{2}$ per nucleon and $R=N C / C C=0.25$. The yield of dimuons is taken as $1 \%$ of CC events over $30 \mathrm{GeV}$... and all of the neutrinos in this dichromatic beam are above $30 \mathrm{GeV}$.

The yield of diffractively produced vector mesons is based primarily on theoretical speculation at this point. However, Barger, Weiler and Phillips(3) estimate that $20 \%$ of the neutrino-nucleon total cross section at $80 \mathrm{GeV}$ could be due 
to production of intermediate vector mesons. If $5 \%$ of such vector mesons decay giving positive muons, this would then account for dimuons at the $1 \%$ level of single muon events. However, Einhorn and Lee ${ }^{(5)}$ give lower estimates of diffractive production for antineutrinos (5\%) and "even less" for neutrinos. Experimental evidence of the diffractive peak in the $x$-distribution is needed. 


\section{DILEPTON PRODUCTION}

The major features of the discovery of dimuons in high energy neutrino interactions have already been discussed, including the intriguing possibilities that a new (and perhaps "charmed") particle, $\mathrm{Y}^{+}$, is produced at the hadronic vertex. Some controversy still exists regarding possible backgrounds, and any new investigation should carefully check the experimental results of the HPWF group. The new experiment should also seek to obtain experimental evidence relating to interesting theoretical suggestions which have been made: for example, are dimuons diffractively produced?

The bubble-chamber technique is well suited to search for diffractive production of vector mesons. In particular, this experiment will be able to examine the diffractive nature of an estimated 100 dimuon events. Both $C C$ and NC events should be analyzable.

Another feature of the heavy liquid bubble chamber/EMI is the ability to search for $e-\mu$ dilepton events. If the decay $\mathrm{Y}^{+} \rightarrow \mathrm{e}^{+}+\nu_{\mathrm{e}}+$ hadrons occurs, $C C$ events will contain $e^{+} \mu$-pairs. Lepton universality requires that $e-\mu$ events should be as common as $\mu-\mu$ events; thus we would expect about $100 \mathrm{e}-\mu$ events. (These are not seen in the present HPWF experiments since electons cannot be recognized easily in the hadron calorimeter.)

Although the arguments given by the HPWF group against 
neutral heavy leptons as a source of dimuons seem persuasive, it is important to be sure that the experimental data used to arrive at these interpretations be checked by a totally different experiment. For example, there may be biasses in $M_{u u}$ invariant mass distribution, the $W_{m i n}$ distribution, or the $\mu^{-}$and $\mu^{+}$distributions from the E-IA geometry and selection criteria. The bubble chamber has a much wider geometrical acceptance, and the ability of EMI-II to detect efficientiy both fast and slow muons makes it possible to examine in detail. the hadronic state associated with dimuons. Hence we feel that this dimuon search, even though limited to about 100 dimuons (and perhaps $100 \mathrm{e}-\mu$ events) will contribute in an important way to the understanding of this fascinating phenomenon. 
IV. DIFFRACTIVE PRODUCTION OF VECTOR PARTICLES

Besides dimuons, the bubble-chamber/EMI configuration being proposed in this experiment should be particularly well suited to the study of diffraction processes.

Theoretically these processes occur when the weak current, charged or neutral, scatters diffractively from the target nucleus or nucleon. The resulting hadron system, minus the target recoil, will have the space-time structure of the weak current. Thus we would expect to see $\rho^{+}$and $A_{1}^{+}$production by the charged current in neutrino interactions reflecting the vector/axia-vector nature of the charged current. The space-time structure of the neutral current is not established, and the study of diffractive processes in muonless neutrino interactions should yield important information to that end.

In addition to the well-known mesons--p, $A_{1}, \omega, \phi$, etc.--one may hope to see evidence for states predicted by charm theories, e.g., $F^{*}, \phi_{C}$, or new unpredicted states coupling to the weak current.

Using vector dominance and the Weinberg-Salam model, Gaillard, Jackson and Nanopoulos (5) have estimated the relative cross sections for the diffraction production of mesons. For the charged currents $\rho^{+}$production is of the order of 5-10\%, $A_{1}^{+}$about $1-2 \%$. Other states--F*, etc.--are predicted to be less than $0.2 \%$. For neutral currents, $A_{1}^{0}$ production 
is $1-2 \%$ with $\rho^{0}$, $\phi$, etc., below $0.2 \%$. The reason for the rather low $\rho^{0}$ production by neutral currents is apparently the result of destructive interference between the weak and electromagnetic currents in the Weinberg-Salam model, where the neutral current is of the form $v_{\mu}-A_{\mu}-2 \sin ^{2} \theta_{W_{\mu}^{j}}{ }_{\mu}^{e} \cdot m$. Thus evidence for $\rho^{0}$ production at the 1 or $2 \%$ level would mitigate against Weinberg-Salam.

other authors have also attempted to estimate the diffraction production of charmed mesons with some disagreement. on results. $(3,4,15)$ Certainiy these calculations are only meant to be crude estimates and should not be taken as final. With the narrow-band beam proposed in this experiment the bulk of the data should be above the thresholds for the predicted states.

Experimentally the bubble chamber allows us to separate the meson system from the target recoil. Furthermore, this meson system will be characterized by high momentum in the diffraction process. Most of the tracks should head downstream in the direction of the EMI. The thicker absorber in the central region will enable a more reliable separation of muons from high momentum hadrons for which punch-through has been a problem with the present EMI. This will be particularly important here since the charged-current diffraction events will be characterized by a slow muon (high $\nu$, low $Q^{2}$ ) and fast hadrons, unlike the bulk of charged current events in which the muon is the highest momentum particle. 
The bubble-chamber mix proposed here also appears to be close to optimum for the study of diffraction processes. The Neon will provide a reasonable event rate and good neutral detection. The latter is especially important if we are to determine the nature of the produced meson system. The 1 ight mix will'give us a reasonable ability to reconstruct the meson system kinematically with good measurements of track momenta. Furthermore, it is more likely for us in the light mix to isolate a subset of events that are produced in hydrogen. For these events there will not be the uncertainty of Fermi momentum, and one could make a more accurate determination of the lepton-neutrino invariant mass in the leptonic or semi-leptonic decay of new hadron resonance. 
V. NEUTRAL CURRENT INTERACTIONS

Although there is a fair understanding of the charged current (single muon) neutrino and antineutrino interactions, based upon both counter and bubble-chamber experiments, the investigation of neutral currents (muoniess interactions) has barely begun. The important discovery of neutral current neutrino interactions by the Gargamelle collaboration has not yet been followed up by any systematic measurement of the Bjorken $x$ and $y$ distributions. In fact, it has been difficult to measure the total yield ratio $R=N C / C C$ accurately due to difficulties in clearly determining whether or not a muon is present. The present counter experiments suffer from a lack of adequate geometrical coverage by their muon spectrometer. It is very difficult to examine hadrons in detail with spark chambers and scintillation counters of large size. In the bubble-chamber experiments to date the mass of the targetliquid has been inadequate for internal muon detection. Even the Phase I. EMI with its "thin" absorber has limitations in separating muonless events from single muon events.

The proposed Phase II EMI, as noted above, will have greatly increased power to distinguish slow muons from slow hadrons. Furthermore, the Internal Picket Fence should reduce the number of "fake muons" by eliminating accidental coincidences. As a separate and longer range project, we propose that additional absorber and a second plane be extended to a full 180 degrees (azimuth) and 60 degrees (vertical). 
Analysis of neutral current events depends upon a precise detailed knowledge of the final hadronic state since the outgoing neutrino cannot be detected. A bubble-chamber experiment has many advantages over counters in this regard provided that neutral hadrons can be detected with good efficiency. In $30 \%$ neon-hydrogen mixture only about $5 \%$ of the hadronic energy, on the average, will escape undetected. This is less than the uncertainty in hadron calorimeters now in use. In addition the bubble chamber is unique in providing detailed information on the nature of hadrons involved, the resonant substates formed, etc.

Even if all hadrons are measured precisely, however, the escaping neutrino in neutral current events leads to a $0-C$ fit for the event (assuming that a neutrino escapes). With a dichromatic beam we would have a $1-C$ fit (with quadratic ambiguity) which would enable us to eliminate certain types of background. For events which are consistent with the hypothesis of a final-state neutrino it will be possible to calculate the Bjorken variables $x$ and $y$ for each event.

Neutral weak currents are fundamental to those models which seek to unify the weak and electromagnetic interactions (e.g., the Weinberg-Salam model). The apparent lack of $\Delta S=1$ neutral currents implies a breakdown in the traditional weak interaction universality for neutral currents. The bubble chamber is needed to detect strange particles, and the 15-foot chamber with neon-hydrogen should be effective in finding $\Delta S \neq 0$ 
interactions. New particles (related to dimuons?) might be responsible for the muonless events. In any event it is essential that a detailed study be made of the distribution functions for the muonless events.

In this exposure we anticipate a total yield of about 2500 muonless events. This is adequate to obtain good $x$ and y distributions. In addition it will be very interesting to see which resonant substates (e.g., the delta-1236) are produced by neutral currents and with what amplitudes. 
VI. OTHER PHYSICS ASPECTS OF THE EXPERIMENT

Although the special aspects of this experiment are dimuons, neutral currents and diffractive production of vector mesons, it is the nature of a bubble-chamber experiment that one "sees everything" that is produced. For example, in the course of studying 100 dimuons and perhaps 2500 muonless events, it will be essential to examine carefully nearly 10,000 single muon events in order to establish a separation of $C C$ and NC events. Furthermore, the study of diffractive production will require constructing the $x$ - and $y$-distributions so that the existence of a "diffractive peak" may be established. Therefore, we expect to do a complete bubble-chamber experiment, including:

(a) Charged current events (deep inetastic),

(b) Search for $\Delta S=-\Delta Q$ events (i.e., charm),

(c) Search for resonances in the final state, and undoubtedly some new topic which will arise in the course of preparing for this experiment and analyzing the data. 
VII. RELATIONSHIP TO OTHER NEUTRINO EXPERIMENTS

(A) EMI-II Development. As indicated in a separate memorandum, $(6)$ the proposers of this experiment are willing to lead the further development of the EMI into "Phase II" if supported in this endeavor by Fermilab. This is a natural outgrowth of our neutrino interests, and we wish the EMI-BC hybrid system to succeed in doing exciting neutrino physics. The short-range developments (the IPF and the central section of the second plane) are essential to the physics suggested here. We are willing to continue to be responsible for making these new devices work and to integrate them with the EMI system. Details are listed in a separate facility development proposal. (6)

(B) Other Physics Commitments. All members of this collaboration are deeply involved in the Fermilab bubblechamber neutrino program. We view this as a "second-generation" experiment whose design is based on the preliminary results. of the first genaration experiments with which we are now involved (E-45 and E-28 for the UH and LBL groups).

A related future commitment for the present collaboration is E-388 (antineutrinos in neon-hydrogen with a dichromatic beam). Our arguments in favor of light (i.e., about $30 \%$ ) neon-hydrogen filling lead us to hope that when E-388 is run the increased accelerator beam energy and intensity would permit use of $30 \%$ neon also. 
Other UH-LBL commitments at Fermilab (as "EMI partners" in $E-151$ and $E-172)$ are such that the degree of our participation is flexible--those individuals who really work on the run/analysis will become co-authors. If this proposal is approved, it would become (with E-388) the major neutrino program for our groups in 1976-77. 
1. "Characteristics of Dimuons as Evidence for a New Quantum Number," preprint by Harvard-Pennsylvania-Wisconsin-Fermilab (HPWF) collaboration, , 1975; see also

A. Benvenuti et al., Phys. Rev. Letters $\underline{34}, 597$ (1975);

A. Benvenuti et al., Phys. Rev. Letters 34,419 (1975).

2. "Two Horn Narrow Band Neutrino Beams," by C. Baltay and D. Cohen, Columbia University preprint $($, 1975).

3. "Vector Meson Dominance Calculations for the Anomaly and Dimuon Production," by V. Barger, T. Weiler, and R. J. N. Phillips, University of Wisconsin report C00-456 (May 1975).

4. "Contributions of Vector-Meson-Dominance to Charmed Meson Production in Inelastic Neutrino and Antineutrino Interactions," by M. B. Einhorn and B. W. Lee, Fermilab Pub-75/56-THY (JuTy 1975).

5. "Diffractive Elastic Neutrino-Production of Vector Mesons," by M. K. Gaillard, S. A. Jackson, and D. V. Nanopoulos, CERN report TH-2049-CERN (24 July 1975).

6. "EMI-II, A Proposed Improvement Program for the Fermilab External Muon Identifier," by members of the Hawaii-LBL collaboration, Fermilab Technical Memo, TM- (October 1975).

7. Rapporteur talk by Carlo Rubbia at the Stanford LeptonPhoton Symposium (August 1975); also "Multi-Lepton Final States in Neutrino Interactions," by HPWF collaboration, talk presented by Don Reeder at Ohio State Neutrino Workshop (September 3-13, 1975). 
9. NAL Proposal 9 "Proposal for a High Energy Neutrino Experiment in the NAL $30 \mathrm{~m}^{3} \mathrm{H}_{2}, \mathrm{D}_{2}$ Bubble Chamber, "by HawaiiNorthwestern-LBL collaboration (June 1970); replaced by NAL Proposal 9-B, "Proposal to Study Neutrino Interactions in the $30 \mathrm{~m}^{3}$ NAL Bubble Chamber with External Muon Identifier," Hawaii-LBL collaboration (July 9, 1971). The twoplane, 111 ton stainless steel external absorber of Proposal 9 EMI was replaced by a one-plane "Phase I EMI" in Proposal 9-B. Rejection of $94 \%$ pions and acceptance of $96 \%$ muons was predicted for the Phase I EMI with 3.2 collision lengths of internal absorber.

10. "Muon Identification Using Multiwire Proportional Chambers," by F. A. Harris S. I. Parker, V. Z. Peterson, D. E. Yount, and M. L. Stevenson, Nuclear Instruments and Methods $103,345(1972)$.

11. EMI group internal memo, LBL Physics Note 802 , "The Angular Distribution of Particles Striking the EMI Proportional Chambers and the Case for a Two-Plane EMI," by M. L. Stevenson (June 30,1975 ).

12. EMI group internal memo UH-Int.14-75, "Hadron Punch-Through Measured in E-45," by M. W. Peters and V. Z. Peterson, October 8, 1975; a1so, EMI group internal memo LBL-Physics Note 811 , "Hadronic Punch-Thru in the External Muon Identifier," by J. Marriner, October 6, 1975.

13. "An External Muon Identifier for Gargamelle at the SPS," CERN proposal by CERN-ECole Polytechnique group, report 
TC-L/Int. 74-7 (November 22, 1974). Fig. 3.6 of this report summarizes the Monte Carlo predictions of punch-through of hadrons.

14. Table of "Atomic and Nuclear Properties," p. 74 of the "blue book" or "Particle Properties" (April 1974 edition). The absorbers involved in the Fermilab EMI are stainless steel, copper and zinc, whose important properties are:

$\begin{array}{llll} & \text { Stee } & \text { Copper } & \text { Zinc } \\ \text { Density }\left(\mathrm{gm} / \mathrm{cm}^{3}\right) & 7.87 & 8.96 & 7.14\end{array}$

Nuclear absorption

$\begin{array}{rrrr}\text { 1ength, } \lambda_{\mathrm{a}}\left(\mathrm{gm} / \mathrm{cm}^{2}\right) & 135 & 133 & 133 \\ (\mathrm{~cm}) & 17.1 & 14.8 & 18.6\end{array}$

"Collision Lengths" (which include elastic scattering) are not relevant. Pion interaction lengths are about $15 \%$ 1onger than $\lambda_{a}$, while proton interaction lengths are about $20 \%$ shorter. In order to avoid confusion we will express all absorber thicknesses either in $\mathrm{gm} / \mathrm{cm}^{2}$ or terms of $\lambda_{\mathrm{a}}=135 \mathrm{gm} / \mathrm{cm}^{2}$.

15. "A Quark Description of Currents and Diffractive Contributions to Leptoproductions", by Jiunn-Ming Wang and Ling-Lie Wong, BNL-20349 (July, 1975).

16. "The "Internal Picket Fence": A Device that could Reduce the Background in the Phase I External Muon Identifier", by M. L. Stevenson, LBL Physics Note 809 (Oct., 1975). 
Figures

Fig. 1 Present EMI absorber and MWPC detector-plane configuration on 15 -foot bubble chamber.

(a) Elevation view, showing coils and zinc absorber (b) Plan view (two tiers of MWPC shown)

Fig. 2 scatterplot of neutrino event charged secondary track projections onto the existing EMI "plane" (each module = $\left.1 \mathrm{~m}^{2}\right)$. Actual "hits" from $C C$ events from $E-45$ data. A11 tracks with $\mathrm{p} \geq 10 \mathrm{GeV} / \mathrm{c}$ from events in $11 \mathrm{~m}^{3}$ fiducial volume are shown.

Fig. 3 Proposed additional absorber and partial second plane for EMI-II (Phase II EMI). The $3 \mathrm{~m} \times 4 \mathrm{~m} \times 600 \mathrm{gm} / \mathrm{cm}^{2}$ zinc absorber would intercept all hadrons (muons) above $10 \mathrm{GeV} / \mathrm{c}$.

Fig. 4 Sketch showing outline E-IA target-calorimeter (dotted lines) superimposed on the outline of the 15-foot bubble chamber.

Fig. 5 Sketch (elevation view) of Internal Picket Fence counters inside 15-foot bubble-chamber coils.

Fig. 6 Two-horn dichromatic neutrino beam spectrum $(100 \pm 5$ GeV/c meson parents, $300 \mathrm{GeV}$ protons). From Ref. 2. 

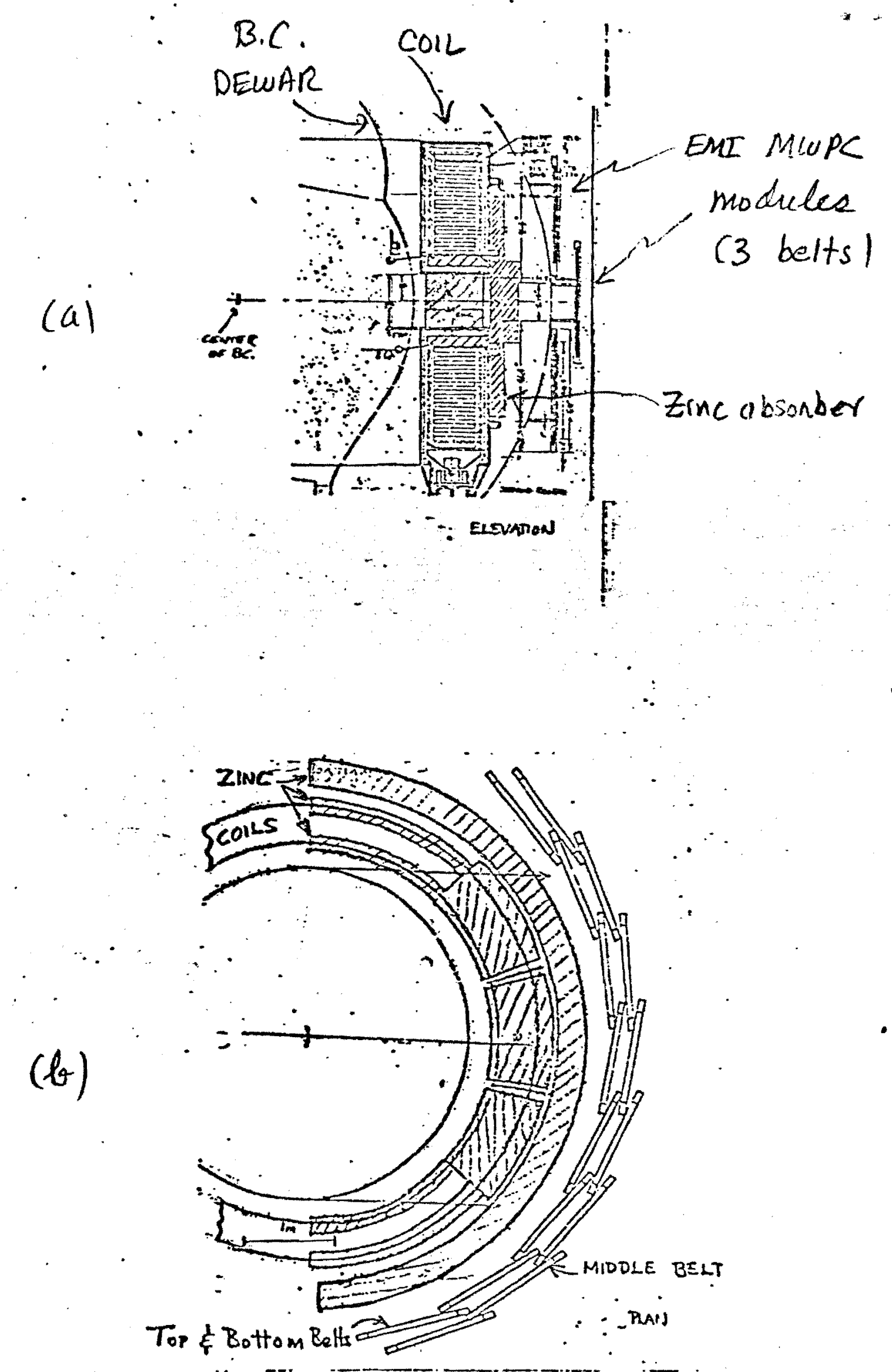

FFG. 1 


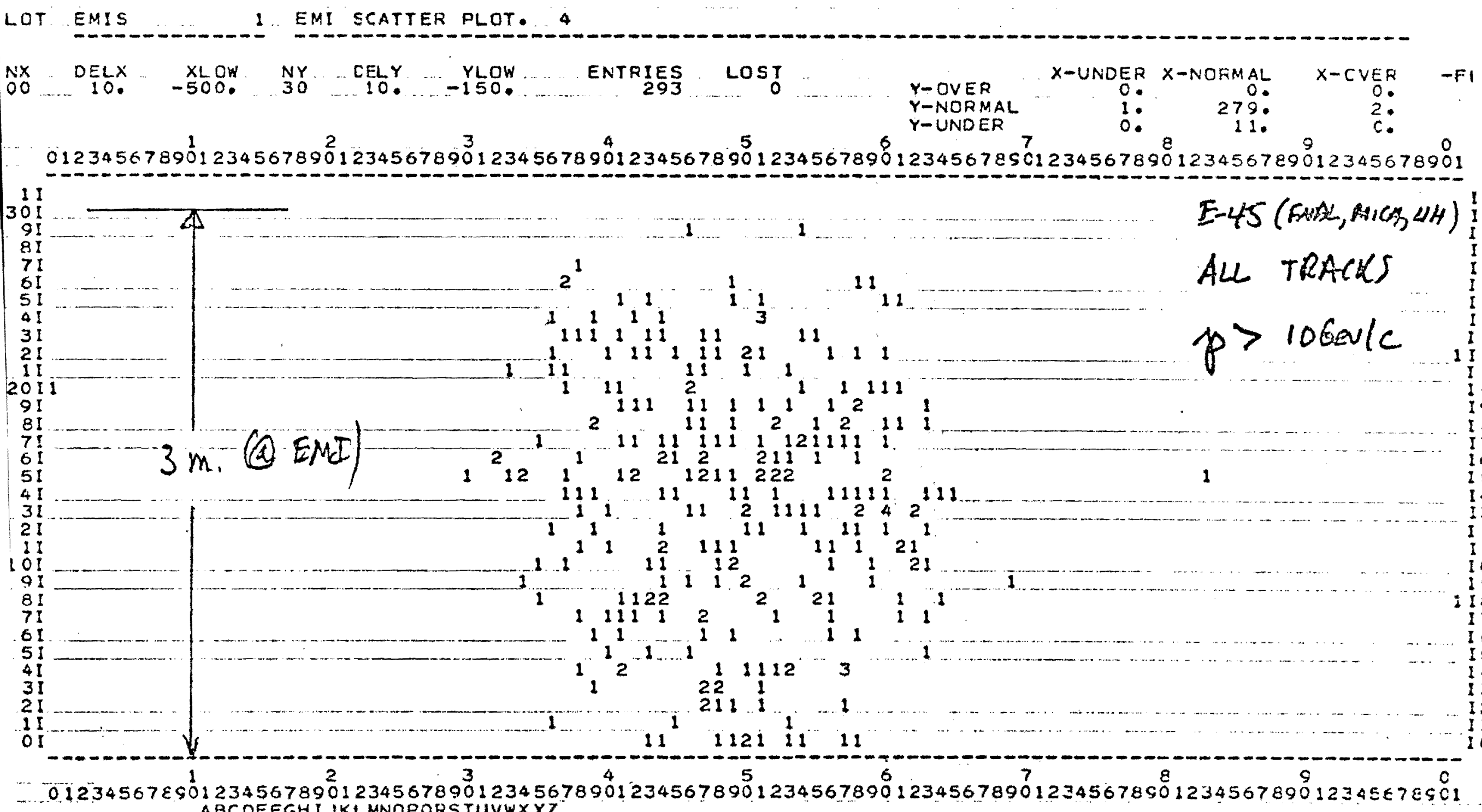

$\Lambda$

$F / G, 2$ 


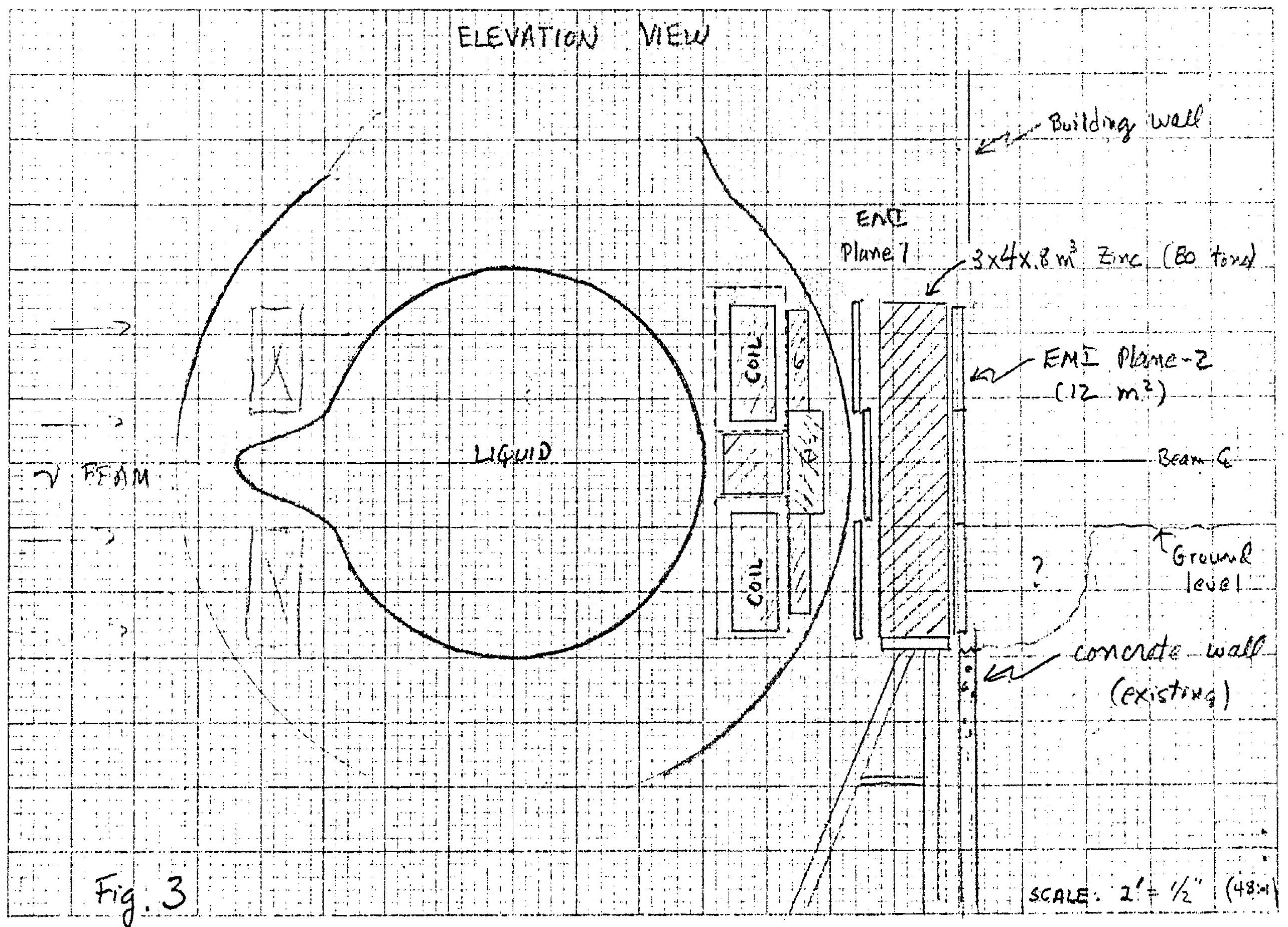


Comparison of Geometxichl Actemtanes E-IA and 15'BC/ENT

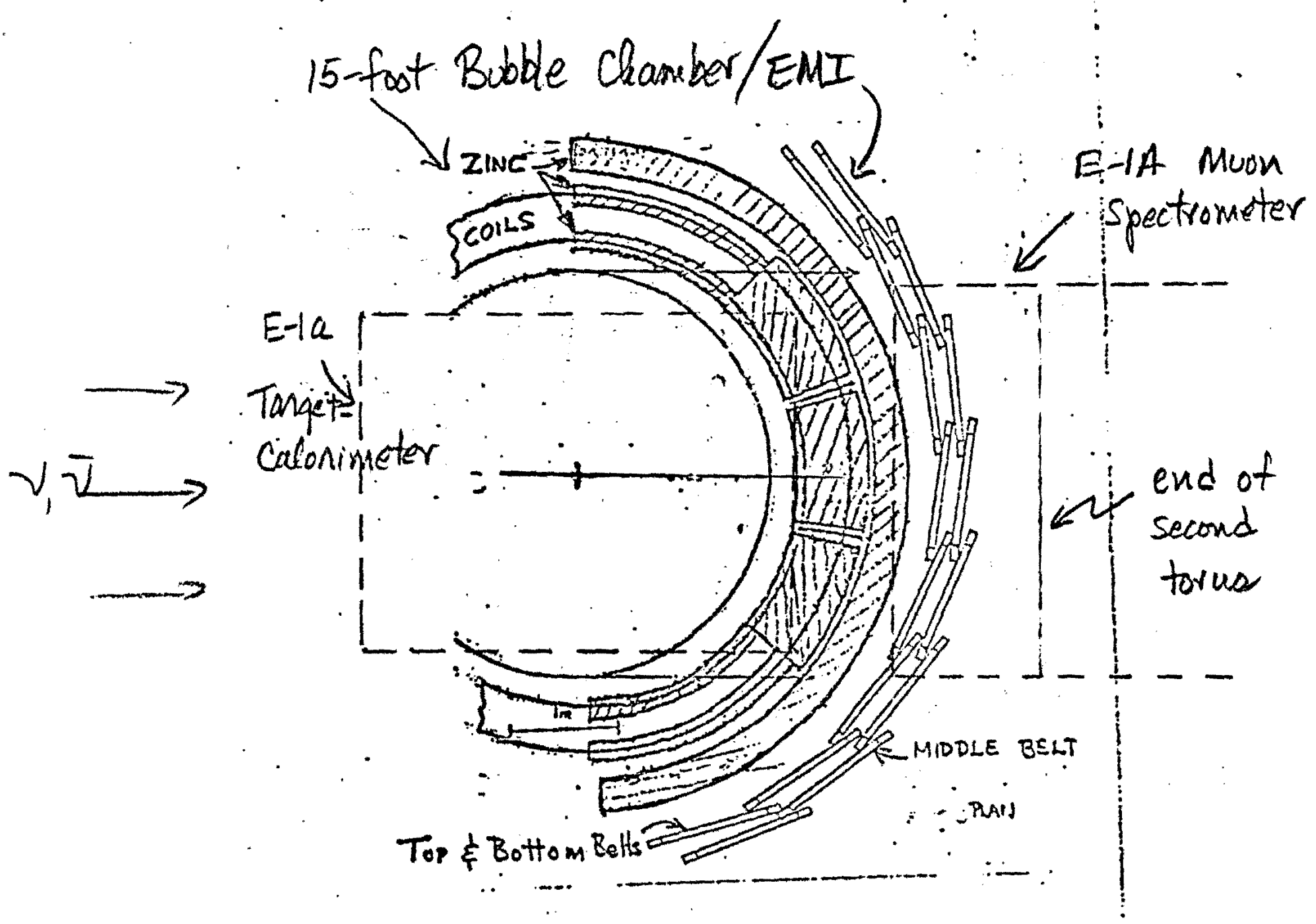

Frg. 4 
DNTERUAL PICKET feme

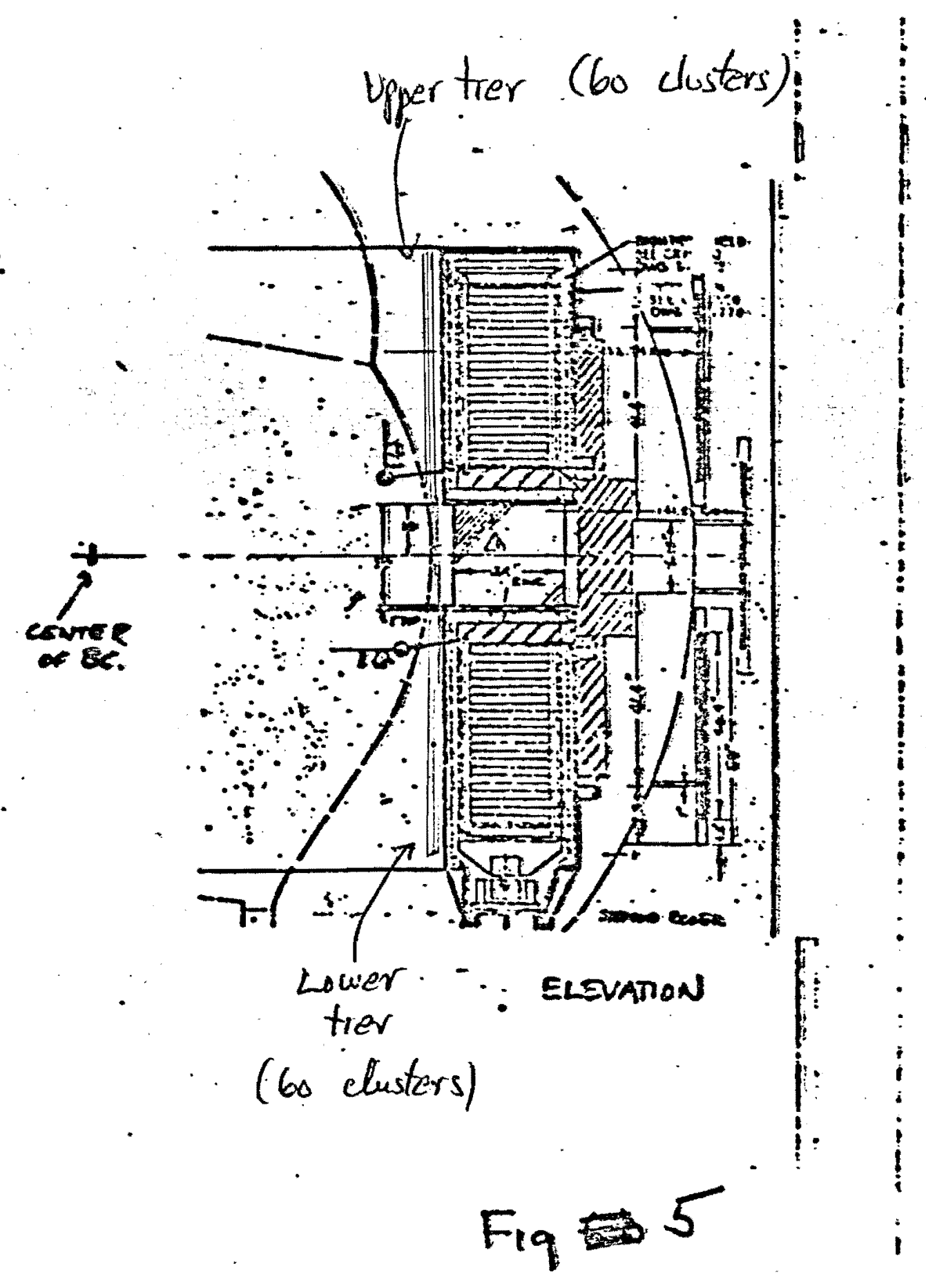

TI: 
